\title{
2 Origami Needle Guide for CT-Guided Interventions
}

\author{
Austin Taylor ${ }^{1}$, Sheng Xu ${ }^{2}$, Bradford J. Wood ${ }^{2}$ and Zion Tsz Ho Tse 1,* \\ 1 School of Electrical and Computer Engineering; austintaylor@uga.edu \\ 2 National Institutes of Health \\ * Correspondence: ziontse@uga.edu; Tel.: 617-838-7006
}

\begin{abstract}
The objective of this study is to preliminarily evaluate a new CT-biopsy guidance device, an origami needle guide. The device is created by laser cutting the structure from a sheet of cardboard, 3D printing two radiocontrast agent grids on to the surface and folding the structure into a rectangular prism with a viewing window. An abdominal imaging phantom was used to evaluate the device through CT imaging and the targeting of lesions for needle insertion. The lesion targeting trials resulted in a mean targeting error of $1.88 \mathrm{~mm}$ with a standard deviation of $0.73 \mathrm{~mm}$. The device attaches to the patient and is rigid enough to adequately support standard biopsy needles, reducing the effect of gravity and the risk of laceration by the needles, making it potentially advantageous for biopsy of superficial lesions and lesions approached from a horizontal orientation. The device supports insertion of multiple needles at once, making it particularly suitable for composite ablation using multiple needles. Another advantage of the device is that it can guide offaxial needle insertion. The low-cost and disposability of the device make it well-suited for the minimally invasive image guided therapy environment.
\end{abstract}

Keywords: origami; percutaneous biopsy; computed tomography; radiologic phantom; 3D printing

\section{Introduction}

Image-guided percutaneous biopsy has become an essential practice in modern medical care. Its safety and efficacy have been verified in numerous studies [1-5]. Nevertheless, various complications still arise, including hemorrhaging, hematomas, and injury to surrounding anatomical structures [611]. The risk of complications may be amplified if the lesion location is difficult to reach or if patient positioning is suboptimal.

Traditionally, CT-guided procedures are performed using the freehand technique. This can be time consuming, especially if the target is small or difficult to reach. In these scenarios, numerous needle insertions and CT scans may be required, thus increasing time, risk, needle manipulations, tissue damage and radiation dosage. The development of guidance systems has improved the safety and efficacy of image-guided interventional procedures. Various laser guidance systems have been developed and implemented [12-14]. However, these systems require the needle to stay axially aligned with the laser beam throughout the insertion and do not provide physical support to hold the needle in place. Other guidance systems have aimed to address these issues but can only accommodate a single needle [15-22].

The origami needle guide is an external localization device intended to address the limitations of previous guidance systems. The device consisted of a cardboard structure created by folding an origami pattern, two layers of a radiocontrast agent (RCA) grid, and a window for viewing the needle insertion. Two-sided adhesive tape applied to the bottom of the device was used to mount the device securely to the patient. When the desired angle of approach had been determined from the CT images, the RCA gridlines provided valuable points of reference to act as a guide for precise localization of lesions.

The device created several potential benefits for CT-guided percutaneous biopsy. First, since the device is attached to the patient, it effectively loosely holds biopsy needles in place during imaging. This is accomplished with a loose needle grip that reduces the risk of tissue laceration when the 
physician needs to let go of the needles. This could be useful for superficial lesions, lesions in which a horizontal approach is required, or for heavier or longer needles, where gravity requires needle stabilization. Second, the device allows for multiple needles to be inserted, making it particularly suitable for composite ablation using multiple needles. Finally, in highly inaccessible lesions that require multiple plane angulations, the device may improve access to the target by allowing off-axial needle insertion. This study aims to describe a novel, low-cost, and disposable device which might provide a cost-effective method for holding and guiding a biopsy needle to a target location during CT guided interventions.

\section{Materials and Methods}

\subsection{Origami Needle Guide Design}

The design of the origami needle guide originated from specifications suggested by surgeons who operate the device as well as the MRI interventional radiologists. Suggestions included:

- Disposable or able to be sterilized for future use.

- Attached securely to patient.

- Allow for in-plane or out of plane needle insertions.

- Support insertion of multiple needles.

- The device should not require additional software.

A template-based guidance system was determined to be the most effective way to satisfy the suggested design specifications. The origami needle guide (Figure 1a) is constructed by laser cutting an origami folding pattern (Figure $1 \mathrm{~b}$ ) into a cardboard material, 3D printing two RCA grids (Figure 1c) onto opposite sides of the folding pattern and folding the device into its final form. The folding pattern is designed with computer-aided design (CAD) software, AutoCAD, and cut with a Full Spectrum laser cutter. The folding pattern design consists of five panels which fold to form a $50 \mathrm{~mm}$ x $100 \mathrm{~mm} \times 100 \mathrm{~mm}$ rectangular prism with one side left open to serve as a viewing window. The folded panels are held in place with eight tabs which fit snuggly into designated slots.

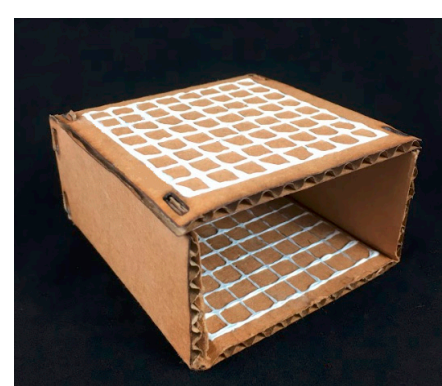

(a)

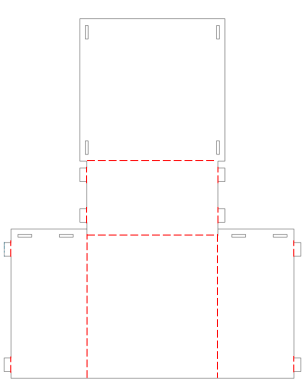

(b)
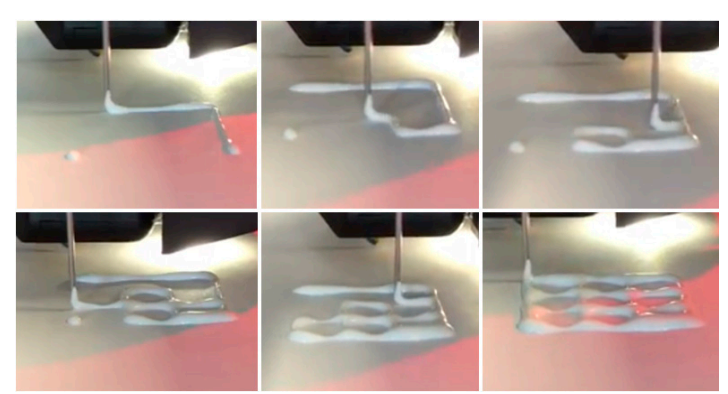

(c)

Figure 1. (a) Origami needle guide. (b) Needle guide folding pattern; solid black lines represent cut lines and dashed red lines represent fold lines. (c) Printing RCA mixture.

\subsection{Radiocontrast Agent Mixture}

The RCA mixture was developed with three major design criteria in mind. The first and most important goal was for the mixture to be bright enough to show up in a CT scan. Secondly, the mixture needed to be applied to a surface as a liquid and be able to dry into a solid. Lastly, we designed the RCA mixture to have a viscosity which would allow it flow through a $5 \mathrm{~mm}$ orifice for extrusion via a syringe in a controlled manner. The resulting mixture was two parts Elmer's glue, one part barium sulfate, and one part water. Barium sulfate, Hi-LR from HiMedia Laboratiories was chosen as the radiocontrast agent due to its exceptional radiopacity [23]. 


\subsection{D Printing Radiocontrast Agent}

To increase manufacturability and to decrease fabrication time, the RCA mixture grid was 3D printed onto the surface of the device. A Fisher Scientific syringe pump was used to extrude the mixture from an EXELINT $50 \mathrm{ml}$ Luer Lock Tip syringe at a constant rate. A $5 \mathrm{~mm}$ diameter tube connected the syringe to the side of an extruder head of a MakerBot 3D printer. The mouth of the tube was aligned vertically with the 3D printer nozzle so that both contacted the printing surface at the same time. A 3D printing job was run on the printer which moved the extruder head along the path of the desired grid. The syringe pump was manually turned on and off when the print job started and finished.

\subsection{Lesion Targeting Equations}

Figure 2 depicts a 2D schematic diagram of a needle trajectory through the device to a target lesion. From the schematic, several equations can be derived to determine the proper coordinates $\left(x_{1}\right.$, $\left.y_{1}, x_{2}, y_{2}\right)$ in the top and bottom layers of the device through which to insert the needle to hit the target lesion. Table 1 defines the variables used in the targeting equations. From a CT scan, the physician can determine the desired insertion angle $\theta$ and the approximate width, length, and depth $\left(T_{x}, T_{y}, T_{z}\right)$ the target is from the origin of the device. Given the target depth $T z$, the vertical distance from the target layer to the bottom layer $z_{2}$ can be found by

$$
z_{2}=T_{z}-z_{1}
$$

Knowing the insertion angle, the horizontal distance from the second insertion location to the target $x_{b}$ can be found by

$$
x_{b}=\frac{z_{2}}{\tan \theta}
$$

The target width $T_{x}$ is the sum of the horizontal distance from the origin to the insertion location on the bottom layer $x_{2}$ and the horizontal distance from the bottom layer insertion location to the target $x_{b}$, so $x_{2}$ can be found by

$$
x_{2}=T_{x}-x_{b}
$$

The horizontal distance from the origin to the top layer insertion location can be found in a similar manner by

$$
\begin{gathered}
x_{a}=\frac{z_{1}}{\tan \theta} \\
x_{1}=x_{a}-x_{2}
\end{gathered}
$$

The insertion coordinates $y 1$ and $y 2$ can be found using the same equations, replacing $x$ with $y$ and $T_{x}$ with $T_{y}$. The insertion depth can be found by

$$
d=\sqrt{\left(x_{a}+x_{b}\right)^{2}+\left(z_{1}+z_{2}\right)^{2}}
$$

\begin{tabular}{cc} 
& Table 1. Variables in needle insertion location equations $(1)-(6)$. \\
\hline Variable & Description \\
\hline$T$ & Target point; $T=\left(T_{x}, T_{y}, T_{z}\right)$ \\
$O$ & Origin of device \\
$\theta$ & Insertion angle \\
$x_{1}, x_{2}$ & Horizontal distance from origin to insertion locations \\
$z_{1}, z_{2}$ & Vertical distance from top layer to bottom layer, vertical distance from bottom \\
& layer to target \\
\hline
\end{tabular}



Needle insertion depth

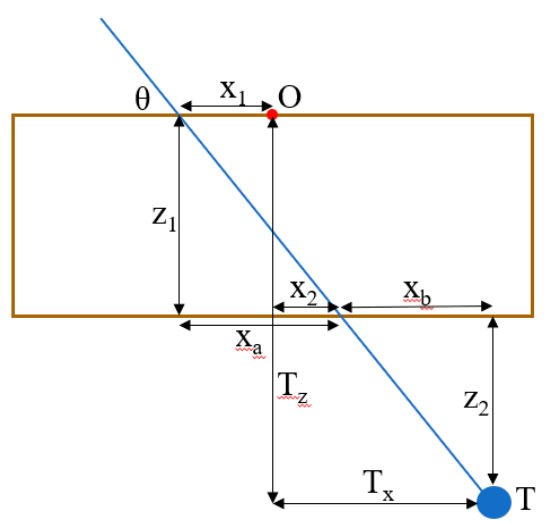

Figure 2. Schematic diagram of a needle insertion trajectory.

\section{2.5. Workflow}

The workflow of a typical procedure using the device is described below.

1. Perform a diagnostic CT or ultrasound scan of the target area to locate the target lesion to determine positioning of the patient and the approximate skin entry point or region.

2. Place the needle guide on the patient and perform another CT scan to visualize the location of the target lesion with respect to the needle guide.

3. Measure the approximate transverse, axial, and sagittal distances from the origin of the needle guide to the target lesion on the CT console or workstation.

4. Use the needle insertion location equations (1) - (6) to determine the insertion locations and the insertion depth. (This step may be semi-automated)

5. Insert the needle into the calculated locations of the needle guide, stopping insertion just after traversing the skin.

6. Perform another CT scan in the same respiratory cycle to confirm the needle is aligned with the target lesion. If yes, continue to step 7. If no, repeat steps $3-6$.

7. Continue pushing the needle the entire calculated insertion depth to contact the target lesion.

8. Perform another CT scan to confirm the target lesion is on track to be sampled (depending upon forward throw gun versus one snap gun). If yes, collect sample and remove the needle from the patient. If no, retract the needle and repeat steps $6 \& 7$.

\subsection{Validation of Targeting Accuracy}

An abdominal phantom was used to perform lesion-targeting experiments $(n=30)$ to validate the accuracy of the needle guide (Figure 3a). The phantom consisted of a 3D printed outer shell with a soft plastic filling designed to match the density of human fatty tissue. The phantom contained various soft 3D printed tumors located throughout the abdominal cavity which were used as targets for needle insertions. The needle guide was positioned in the ventral insertion window of the phantom and the origin of the needle guide was aligned with the CT laser to assure proper craniocaudal angulation. After initial scans, the insertion locations and the insertion depth were calculated, and the needle was inserted (Figure 3b). Using confirmation scans, transverse and sagittal distance errors can be calculated based on the distance between the needle tip and the target tumor

141 (Figure 3c). Total error is calculated as the hypotenuse of the transverse and sagittal errors. 


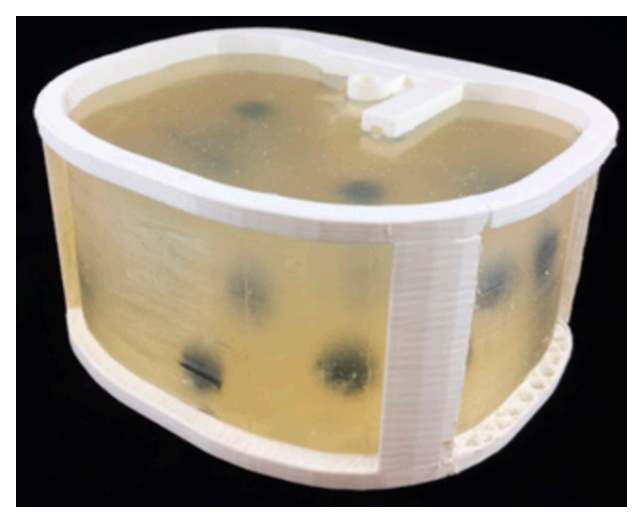

(a)

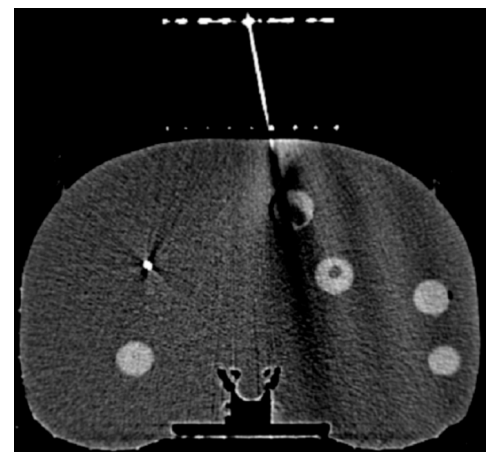

(c)

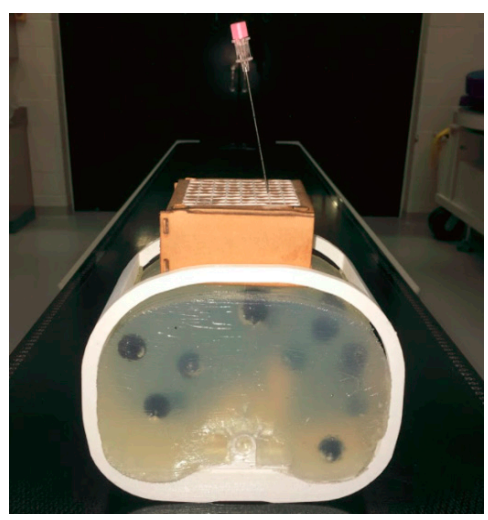

(b)

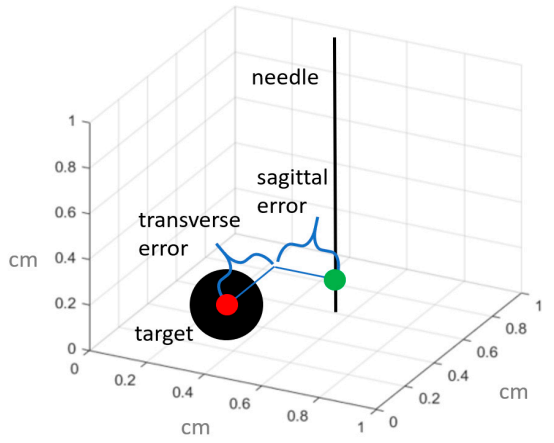

(d)

Figure 3. (a) 3D printed abdominal phantom for quantification of the lesion targeting error. (b) Origami needle guide mounted to phantom. (c) CT image showing needle insertion. (d) Targeting errors were measured in both transverse and sagittal directions of the needle relative to the target lesion.

\section{Results}

\subsection{Workspace Analysis}

Figure 4 shows the possible needle trajectories in the transverse plane provided by the gridlines on the origami needle guide. The discrete potential insertion locations correspond to RCA gridlines as well as midlines between each of the RCA gridlines, illustrated by a raster engraving from the laser cutter. The RCA grids provides a high density of discrete guidelines, while the puncturable device material offers a continuous workspace for potential needle insertions. 


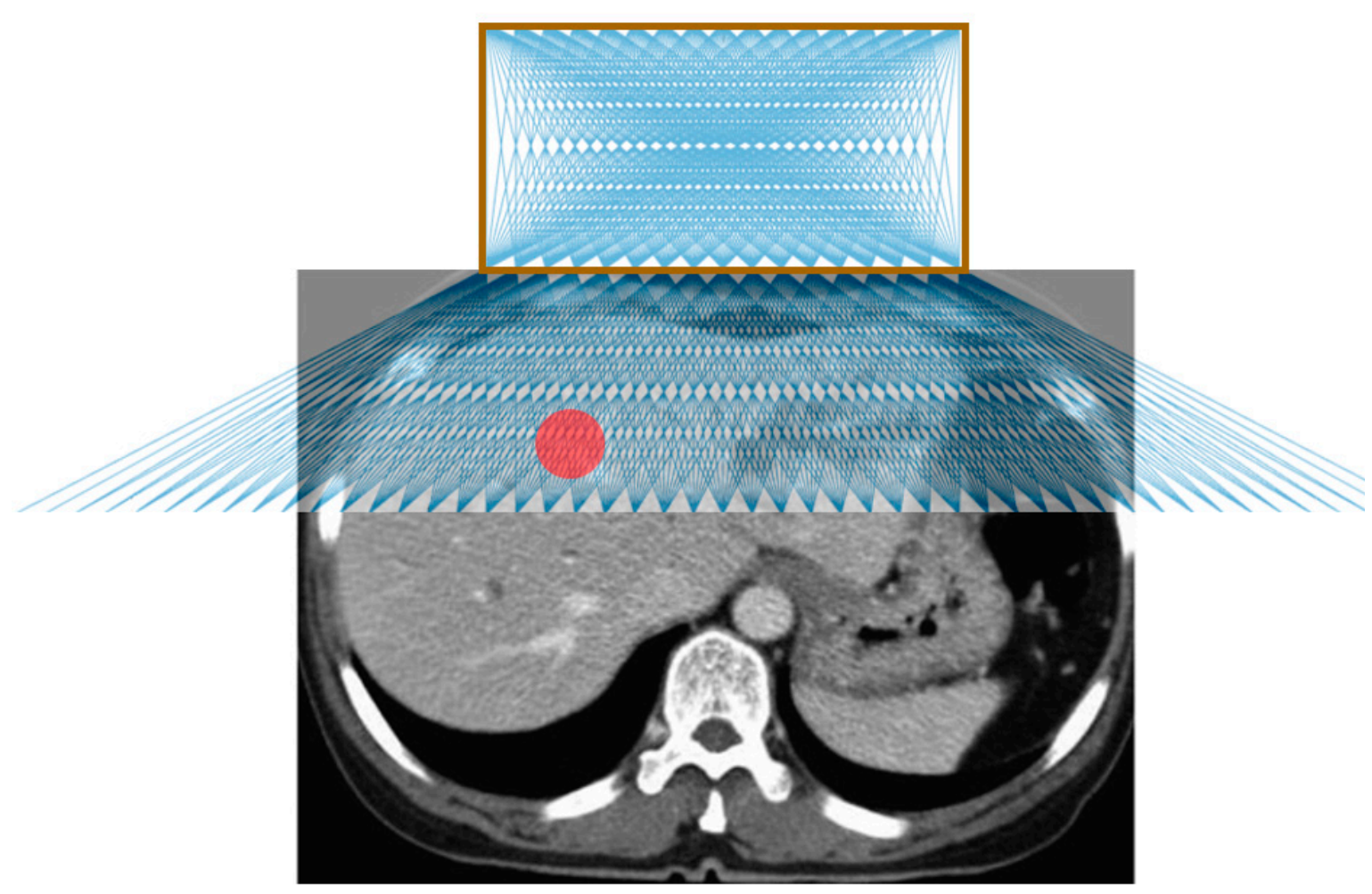

Figure 4. Illustration of possible needle trajectories in the transverse plane.

\section{4}

155

156

157

158

159

160

\subsection{Phantom Study}

The results of the needle insertion experiments performed on an abdominal phantom are displayed in Figure 5. Preliminary results showed successful CT-guided biopsy needle placements in an abdominal phantom. The mean targeting accuracy over all experiments was $1.88 \mathrm{~mm}$ with a standard deviation of $0.73 \mathrm{~mm}$. Figure 5a displays a box and whisker plot comparing the targeting error between the sagittal and transverse axes, and Figure $5 \mathrm{~b}$ depicts the radial error. The results show that the error is relatively evenly distributed around the target.

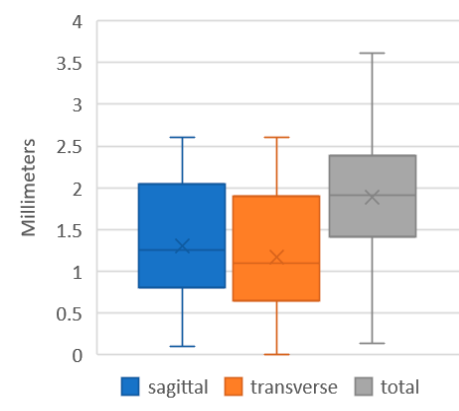

(a)

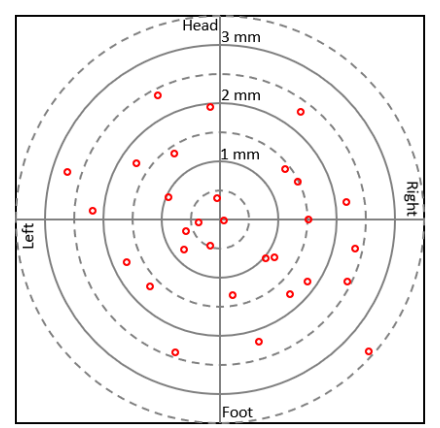

(b)

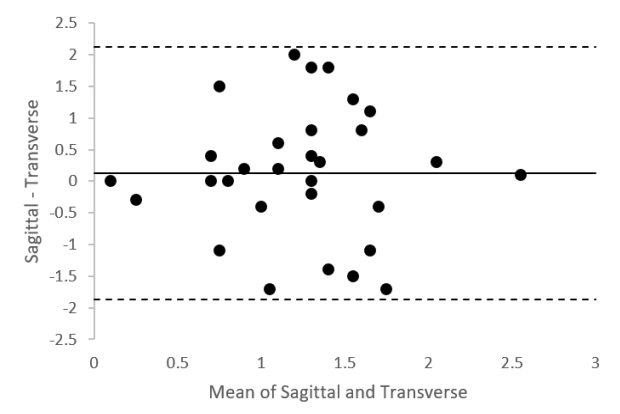

(c)

Figure 5. Plots of the lesion targeting error in phantom studies. (a) Quartile plot of sagittal, transverse and total targeting error. (b) Plot of radial error. Solid rings every millimeter, dashed rings ever 0.5 mm. (c) Bland-Altman plot of sagittal and transverse error measurements.

\section{Discussion}

The origami needle guide is a potentially useful tool for percutaneous biopsy or ablation. Preliminary experiments showed successful lesion targeting in an abdominal phantom. One primary advantage is that since the device is attached to the patient, it holds needles in place, allowing scanning while the needle remains aligned along the desired trajectory, thus potentially reducing the risk of tissue laceration by rigidly held needles. This attribute also makes the device advantageous 
for superficial lesions and lesions that must be approached from a horizontal orientation. Another major benefit is that the device allows for multiple needles to be inserted, making it applicable for composite ablation using multiple needles. One more advantage of the device is that is can guide offaxial needle insertion for highly inaccessible lesions that require multiple plane angulations. Furthermore, the device can be manufactured quickly and inexpensively, making it disposable and therefore ideal for the surgical environment.

Our preliminary evaluation exposed several limiting characteristics. The fixed nature of the needle guide may be disadvantageous in situations where the target lesion moves with time, such as with pulmonary lesions during respiration. Another limitation is that the large footprint of the device can make it difficult to be used with ultrasound. In future work, we aim to manufacture the device with biocompatible or sterilize-able materials. However, the biocompatibility and hemocompatibility regulatory issues of having the needle traverse the material also need to be certified. Given the limitations of our methodology, more rigorous testing in a specific clinical application would be necessary to compare this device with established systems.

\section{Conclusions}

A novel device for assisting with CT-guided needle insertions is presented. The device was fabricated by laser cutting the structure from a sheet of cardboard, 3D printing two radiocontrast agent grids on to the surface and folding the structure into a rectangular prism with a viewing window. The device was evaluated through CT imaging and targeting of lesions for needle insertions in an abdominal imaging phantom. The results of the lesion targeting experiments showed a mean targeting error of $1.88 \mathrm{~mm}$ with a standard deviation of $0.73 \mathrm{~mm}$. The main advantages of the device are that it attaches to the patient (potentially reducing the risk of laceration), it supports insertion of multiple needles (making it particularly suitable for composite ablations), and it can guide off-axial needle insertion. The low-cost and disposability are well-suited for interventional settings.

Author Contributions: Conceptualization, A.J.T. and Z.T.H.T.; methodology, A.J.T.; validation, A.J.T. and Z.T.H.T.; formal analysis, A.J.T.; investigation, A.J.T.; resources, Z.T.H.T.; data curation, A.J.T.; writing - original draft preparation, A.J.T.; writing - review and editing, S.X., B.W., and Z.T.H.T.; visualization, A.J.T.; supervision, Z.T.H.T.; project administration, Z.T.H.T.; funding acquisition, A.J.T. and Z.T.H.T.

Funding: This research was funded by the National Institutes of Health (NIH) Bench-to-Bedside Award, the NIH Center for Interventional Oncology Grant, the National Science Foundation (NSF) I-Corps Team Grant (1617340), NSF REU site program 1359095, the UGA-AU Inter-Institutional Seed Funding, the American Society for Quality Dr. Richard J. Schlesinger Grant, the PHS Grant UL1TR000454 from the Clinical and Translational Science Award Program, the NIH National Center for Advancing Translational Sciences, and the Intramural Research Program of the NIH.

Conflicts of Interest: The authors declare no conflict of interest. The funders had no role in the design of the study; in the collection, analyses, or interpretation of data; in the writing of the manuscript, or in the decision to publish the results. The conclusions are the opinions of the authors and do not reflect those of the US Government which does not endorse products.

\section{References}

1. [1] H. Li, P. M. Boiselle, J. Shepard, B. Trotman-Dickenson, and T. McLoud, "Diagnostic accuracy and safety of CT-guided percutaneous needle aspiration biopsy of the lung: comparison of small and large pulmonary nodules," AJR. American journal of roentgenology, vol. 167, no. 1, pp. 105-109, 1996.

2. [2] L. Whitmire, J. Galambos, V. Phillips, C. Sewell, B. Erwin, W. Torres, R. Gedgaudas-McClees, and M. Bernardino, "Imaging guided percutaneous hepatic biopsy: diagnostic accuracy and safety," Journal of clinical gastroenterology, vol. 7, no. 6, pp. 511-515, 1985.

3. [3] S. G. Leffler and F. S. Chew, "CT-guided percutaneous biopsy of sclerotic bone lesions: diagnostic yield and accuracy," AJR. American journal of roentgenology, vol. 172, no. 5, pp. 1389-1392, 1999.

4. [4] T. Welch, P. Sheedy 2nd, C. Johnson, C. Johnson, and D. Stephens, "CT-guided biopsy: prospective analysis of 1,000 procedures," Radiology, vol. 171, no. 2, pp. 493-496, 1989. 
5. [5] J. R. Haaga and R. J. Alfidi, "Precise biopsy localization by computed tomography," Radiology, vol. 